\title{
BIHARMONIC LAGRANGIAN SURFACES OF CONSTANT MEAN CURVATURE IN COMPLEX SPACE FORMS
}

\author{
TORU SASAHARA \\ Department of Mathematics, Oita National College of Technology, 1666 Maki, Oita 870-0150, Japan \\ e-mail: t-sasa@oita-ct.ac.jp
}

(Received 12 December, 2006; revised 9 May, 2007; accepted 24 May, 2007)

\begin{abstract}
Biharmonic Lagrangian surfaces of constant mean curvature in complex space forms are classified. A further important point is that new examples of marginally trapped biharmonic Lagrangian surfaces in an indefinite complex Euclidean plane are obtained. This fact suggests that Chen and Ishikawa's classification of marginally trapped biharmonic surfaces [6] is not complete.
\end{abstract}

2000 Mathematics Subject Classification. Primary 53C42; Secondary 53B25.

1. Introduction. The study of Lagrangian submanifolds from the Riemannian geometric point of view has been a very active field during the last quarter of the century and many interesting results have been obtained. In particular, minimal Lagrangian submanifolds have attracted considerable attention (see, for example, [5]). It should be noted that if the metric of the ambient space is indefinite, the situation is quite different form the Riemannian case (see, [7], [12] and [17]).

A natural generalization of the class of minimal submanifolds from the variational point of view is the one of biharmonic submanifolds (see, [9]), which was introduced by Eells and Sampson [9]. Thus, it is worthwhile and interesting to investigate biharmonic Lagrangian submanifolds. For recent developments in the study of biharmonic submanifolds see, for example, [2], [3], [10] and [15]. In this paper, biharmonic Lagrangian surfaces of constant mean curvature in complex space forms are classified. In particular, we obtain new examples of marginally trapped Lagrangian surfaces in an indefinite complex Euclidean plane. This implies that the classification of marginally trapped biharmonic surfaces due to Chen and Ishikawa [6] is not complete.

2. Preliminaries. Let $\tilde{M}_{s}^{n}(4 \epsilon)$ be a complex space form of complex dimension $n$ and complex index $s(\geq 0)$. The complex index is defined as the complex dimension of the largest complex negative definite vector subspace of the tangent space. The curvature tensor $\tilde{R}$ of $\tilde{M}_{s}^{n}(4 \epsilon)$ is given by

$$
\begin{aligned}
\tilde{R}(X, Y) Z= & \epsilon\{\langle Y, Z\rangle X-\langle X, Z\rangle Y+\langle Z, J Y\rangle J X \\
& -\langle Z, J X\rangle J Y+2\langle X, J Y\rangle J Z\},
\end{aligned}
$$

where $J$ is the almost complex structure of $\tilde{M}_{s}^{n}(4 \epsilon)$.

Barros and Romero [1] showed that locally any complex space form $\tilde{M}_{s}^{n}(4 \epsilon)$ is isometric holomorphically to $\mathbf{C}_{s}^{n}, \mathbf{C} P_{s}^{n}(4 \epsilon)$ or $\mathbf{C} H_{s}^{n}(4 \epsilon)$ according to whether $\epsilon=0$, $\epsilon>0$ or $\epsilon<0$. 
Let $M$ be a Lagrangian submanifold of complex space form $\tilde{M}_{s}^{n}(4 \epsilon)$. It is clear that the real index of $M$ is $s$. We denote by $\nabla$ and $\tilde{\nabla}$ the Levi-Civita connections on $M$ and $\tilde{M}_{s}^{n}(4 \epsilon)$, respectively. The formulae of Gauss and Weingarten are given by

$$
\begin{gathered}
\tilde{\nabla}_{X} Y=\nabla_{X} Y+h(X, Y), \\
\tilde{\nabla}_{X} \xi=-A_{\xi} X+D_{X} \xi,
\end{gathered}
$$

respectively, where $h, A$ and $D$ are the second fundamental form, the shape operator and the normal connection respectively.

Since $J$ is parallel, we have

$$
\begin{gathered}
D_{X} J Y=J\left(\nabla_{X} Y\right), \\
A_{J Y} X=-J h(X, Y)=A_{J X} Y .
\end{gathered}
$$

Denote by $R$ the Riemann curvature tensor of $M$. Then the equations of Gauss, Codazzi and Ricci are equivalent to

$$
\begin{gathered}
R(X, Y ; Z, W)=\epsilon(\langle X, W\rangle\langle Y, Z\rangle-\langle X, Z\rangle\langle Y, W\rangle)+\left\langle\left[A_{J Z}, A_{J W}\right](X), Y\right\rangle, \\
\left(\bar{\nabla}_{X} h\right)(Y, Z)=\left(\bar{\nabla}_{Y} h\right)(X, Z),
\end{gathered}
$$

where $X, Y, Z, W$ are vectors tangent to $M$, and $\bar{\nabla} h$ is defined by

$$
\left(\bar{\nabla}_{X} h\right)(Y, Z)=D_{X} h(Y, Z)-h\left(\nabla_{X} Y, Z\right)-h\left(Y, \nabla_{X} Z\right) .
$$

The mean curvature vector field $H$ is defined by $H=\frac{1}{n}$ trace $h$. The function $\sqrt{|\langle H, H\rangle|}$ is called the mean curvature.

A vector $X$ is called space-like (resp. time-like) if it satisfies $\langle X, X\rangle>0$ (resp. $\langle X, X\rangle<0)$. A vector $X$ is called light-like if it is nonzero and it satisfies $\langle X, X\rangle=0$. Surfaces in pseudo-Riemannaian 4-manifolds are called marginally trapped (or quasiminimal) if $H$ is light-like at each point on the surface.

The Laplace operator which acts on the sections of $\left.T \tilde{M}_{s}^{n}(4 \epsilon)\right|_{M}$ (resp. normal bundle $\left.T^{\perp} M\right)$ is defined by $\Delta=-\sum_{i=1}^{n}\left\langle e_{i}, e_{i}\right\rangle\left(\tilde{\nabla}_{e_{i}} \tilde{\nabla}_{e_{i}}-\tilde{\nabla}_{\nabla_{e_{i}} e_{i}}\right)$ (resp. $\Delta^{D}=$ - $\left.\sum_{i=1}^{n}\left\langle e_{i}, e_{i}\right\rangle\left(D_{e_{i}} D_{e_{i}}-D_{\nabla_{e_{i}} e_{i}}\right)\right)$, where $\left\{e_{i}\right\}$ is a local orthonormal frame of $M$.

We need the following existence and uniqueness theorem (cf. [7]) for the later use.

THEOREM 1. Let $\left(M_{s}^{n},\langle\cdot, \cdot\rangle\right)$ be an $n$-dimensional simply connected manifold of real index $s$, where $s \in\{0,1\}$. Let $\sigma$ be a symmetric bilinear $T M$-valued form on $M$ satisfying

(1) $\langle\sigma(X, Y), Z\rangle$ is totally symmetric,

(2) $(\nabla \sigma)(X, Y, Z)=\nabla_{X} \sigma(Y, Z)-\sigma\left(\nabla_{X} Y, Z\right)-\alpha\left(Y, \nabla_{X} Z\right)$ is totally symmetric,

(3) $R(X, Y) Z=\epsilon(\langle Y, Z\rangle X-\langle X, Z\rangle Y)+\sigma(\sigma(Y, Z), X)-\sigma(\sigma(X, Z), Y)$.

Then there exists a Lagrangian isometric immersion $x:\left(M_{s}^{n},\langle\cdot, \cdot\rangle\right) \rightarrow \tilde{M}_{s}^{n}(4 \epsilon)$ such that the second fundamental form $h$ satisfies $h(X, Y)=J \sigma(X, Y)$.

THEOREM 2. Let $x^{1}, x^{2}: M \rightarrow \tilde{M}_{s}^{n}(4 \epsilon)$ be two Lagrangian isometric immersions of a connected $n$-manifold with second fundamental forms $h^{1}$ and $h^{2}$, where $s \in\{0,1\}$. If

$$
\left\langle h^{1}(X, Y), J x_{*}^{1} Z\right\rangle=\left\langle h^{2}(X, Y), J x_{*}^{2} Z\right\rangle
$$

for all vector fields $X, Y, Z$ tangent to $M$, there exists an isometry $A$ of $\tilde{M}_{s}^{n}(4 \epsilon)$ such that $x^{1}=A \circ x^{2}$. 
3. Biharmonic maps. Let $M^{m}$ and $N^{n}$ be pseudo-Riemannian manifolds of dimension $m$ and $n$ respectively, and $\phi: M \rightarrow N$ a smooth map. We denote by $\nabla$ and $\tilde{\nabla}$ the Levi-Civita connections on $M$ and $N$ respectively. Then the tension field $\tau(\phi)$ is a section of the vector bundle $\phi^{*} T N$ defined by

$$
\tau(\phi):=\operatorname{tr}\left(\nabla^{\phi} d \phi\right)=\sum_{i=1}^{m}\left\langle e_{i}, e_{i}\right\rangle\left\{\nabla_{e_{i}}^{\phi} d \phi\left(e_{i}\right)-d \phi\left(\nabla_{e_{i}} e_{i}\right)\right\} .
$$

Here $\nabla^{\phi}$ and $\left\{e_{i}\right\}$ denote the induced connection by $\phi$ on the bundle $\phi^{*} T N$, which is the pull-back of $\tilde{\nabla}$, and a local orthonormal frame field of $M$, respectively.

A smooth map $\phi$ is said to be a harmonic map if its tension field vanishes. If $M$ is a Riemannian manifold, then $\phi$ is harmonic if and only if it is a critical point of the energy

$$
E(\phi)=\int_{\Omega} \sum_{i=1}^{m}\left\langle d \phi\left(e_{i}\right), d \phi\left(e_{i}\right)\right\rangle d v_{g},
$$

over every compactly supported region $\Omega$ of $M$.

We define the bitension field

$$
\tau_{2}(\phi):=\sum_{i=1}^{m}\left\langle e_{i}, e_{i}\right\rangle\left\{\left(\nabla_{e_{i}}^{\phi} \nabla_{e_{i}}^{\phi}-\nabla_{\nabla_{e_{i}} e_{i}}^{\phi}\right) \tau+R^{N}\left(\tau, d \phi\left(e_{i}\right)\right) d \phi\left(e_{i}\right)\right\},
$$

where $R^{N}$ is the curvature tensor of $N$. We say that a smooth map $\phi$ is a biharmonic map if its bitension field vanishes. Harmonic maps are clearly biharmonic. Non-harmonic biharmonic maps are called proper biharmonic maps. When $M$ is a Riemannian manifold, the biharmonic map $\phi$ is characterized as a critical point of the bienergy

$$
E_{2}(\phi)=\int_{\Omega}\langle\tau(\phi), \tau(\phi)\rangle d v_{g}
$$

over every compactly supported region $\Omega$ of $M$. then

In the case that $N$ is the pseudo-Euclidean space and $\phi$ is an isometric immersion,

$$
\tau_{2}(\phi)=\Delta_{M} \Delta_{M} \phi
$$

since $\Delta_{M} \phi=-m H$. Here $\Delta_{M}$ is the Laplacian acting on $C^{\infty}\left(M^{m}\right)$. Thus the biharmonicity for an isometric immersion into the pseudo-Euclidean space is equivalent to the biharmonicity in the sense of Chen (see [6]).

For recent results about biharmonic maps we refer to the survey [13].

4. Biharmonic Lagrangian surfaces. In this section, we mainly consider proper biharmonic Lagrangian surfaces in $\tilde{M}_{1}^{2}(4 \epsilon)$. In the case that the ambient space is positive definite, i.e., $s=0$, we obtain similar results (see Theorem 11).

Let $M$ be a Lagrangian surface in $M_{1}^{2}(4 \epsilon)$. By (2.5) the second fundamental form takes the form

$$
\begin{gathered}
h\left(e_{1}, e_{1}\right)=a J e_{1}+b J e_{2}, \\
h\left(e_{1}, e_{2}\right)=-b J e_{1}+c J e_{2}, \\
h\left(e_{2}, e_{2}\right)=-c J e_{1}+d J e_{2},
\end{gathered}
$$


for some functions $a, b, c, d$, with respect to an orthonormal frame $\left\{e_{1}, e_{2}\right\}$ such that $\left\langle e_{1}, e_{1}\right\rangle=1$ and $\left\langle e_{2}, e_{2}\right\rangle=-1$. In the rest of this paper we will use this frame.

By differentiating the second fundamental form covariantly we get

$$
\begin{aligned}
& \left(\bar{\nabla}_{e_{2}} h\right)\left(e_{1}, e_{1}\right)=\left(e_{2} a+3 b \omega_{1}^{2}\left(e_{2}\right)\right) J e_{1}+\left(e_{2} b+(a-2 c) \omega_{1}^{2}\left(e_{2}\right)\right) J e_{2}, \\
& \left(\bar{\nabla}_{e_{1}} h\right)\left(e_{1}, e_{2}\right)=-\left(e_{1} b+(a-2 c) \omega_{1}^{2}\left(e_{1}\right)\right) J e_{1}+\left(e_{1} c-(2 b+d) \omega_{1}^{2}\left(e_{1}\right)\right) J e_{2}, \\
& \left(\bar{\nabla}_{e_{1}} h\right)\left(e_{2}, e_{2}\right)=\left(-e_{1} c+(2 b+d) \omega_{1}^{2}\left(e_{1}\right)\right) J e_{1}+\left(e_{1} d-3 c \omega_{1}^{2}\left(e_{1}\right)\right) J e_{2}, \\
& \left(\bar{\nabla}_{e_{2}} h\right)\left(e_{1}, e_{2}\right)=-\left(e_{2} b+(a-2 c) \omega_{1}^{2}\left(e_{2}\right)\right) J e_{1}+\left(e_{2} c-(2 b+d) \omega_{1}^{2}\left(e_{2}\right)\right) J e_{2},
\end{aligned}
$$

where $\omega_{i}^{j}\left(e_{k}\right)=\left\langle\nabla_{e_{k}} e_{i}, e_{j}\right\rangle\left\langle e_{j}, e_{j}\right\rangle$. Note that $\omega_{1}^{2}=\omega_{2}^{1}$. From (2.7) we obtain

$$
\begin{gathered}
e_{2} a+3 b \omega_{1}^{2}\left(e_{2}\right)=-e_{1} b-(a-2 c) \omega_{1}^{2}\left(e_{1}\right), \\
e_{2} b+(a-2 c) \omega_{1}^{2}\left(e_{2}\right)=e_{1} c-(2 b+d) \omega_{1}^{2}\left(e_{1}\right), \\
e_{1} d-3 c \omega_{1}^{2}\left(e_{1}\right)=e_{2} c-(2 b+d) \omega_{1}^{2}\left(e_{2}\right) .
\end{gathered}
$$

The Gauss equation (2.6) yields that the Gauss curvature $G$ is given by

$$
G=a c+b^{2}+b d-c^{2}+\epsilon .
$$

It follows from (2.1) and (3.1) that $M$ is biharmonic if and only if

$$
\Delta H=5 \epsilon H .
$$

By the same computation in [4], we have

$$
\Delta H=\Delta^{D} H+\sum_{i=1}^{2} g\left(e_{i}, e_{i}\right) h\left(e_{i}, A_{H} e_{i}\right)+\operatorname{tr}\left(\bar{\nabla} A_{H}\right),
$$

where $\operatorname{tr}\left(\bar{\nabla} A_{H}\right)=\sum_{i=1}^{2} g\left(e_{i}, e_{i}\right)\left(A_{D_{e_{i}} H} e_{i}+\left(\nabla_{e_{i}} A_{H}\right) e_{i}\right)$.

From now on, we assume that the mean curvature function is constant.

Case (I): $\langle H, H\rangle>0$. In this case, $b=d$. From (4.6) and (4.8) we obtain

$$
e_{2}(a+c)=-(a+c) \omega_{1}^{2}\left(e_{1}\right)
$$

Since $\langle H, H\rangle=\frac{(a+c)^{2}}{4}=$ constant, $\omega_{1}^{2}\left(e_{1}\right)=0$ by (4.12). Hence, (4.6)-(4.8) is reduced to

$$
\begin{gathered}
e_{2} b+(a-2 c) \omega_{1}^{2}\left(e_{2}\right)=e_{1} c \\
e_{1} b=e_{2} c-3 b \omega_{1}^{2}\left(e_{2}\right) .
\end{gathered}
$$

Let $(\Delta H)^{\top}$ be the component of $\Delta H$ tangential to $M$. Put $A_{J e_{i}}=A_{i}$. Then

$$
\begin{aligned}
\frac{1}{|H|}(\Delta H)^{\top}= & A_{D_{e_{1}} J e_{1}} e_{1}+\nabla_{e_{1}}\left(A_{1} e_{1}\right)-A_{1}\left(\nabla_{e_{1}} e_{1}\right)-A_{D_{e_{2}} J e_{1}} e_{2}-\nabla_{e_{2}}\left(A_{1} e_{2}\right)+A_{1}\left(\nabla_{e_{2}} e_{2}\right) \\
= & \nabla_{e_{1}}\left(a e_{1}+b e_{2}\right)-\omega_{1}^{2}\left(e_{2}\right) A_{2} e_{2}-\nabla_{e_{2}}\left(-b e_{1}+c e_{2}\right)+\omega_{2}^{1}\left(e_{2}\right) A_{1} e_{1} \\
= & \left(e_{1} a\right) e_{1}+\left(e_{1} b\right) e_{2}-\omega_{1}^{2}\left(e_{2}\right)\left(-c e_{1}+b e_{2}\right)+\left(e_{2} b\right) e_{1}+b \omega_{1}^{2}\left(e_{2}\right) e_{2} \\
& -\left(e_{2} c\right) e_{2}-c \omega_{2}^{1}\left(e_{2}\right) e_{1}+\omega_{2}^{1}\left(e_{2}\right)\left(a e_{1}+b e_{2}\right) \\
= & \left\{-e_{1} c+e_{2} b+a \omega_{1}^{2}\left(e_{2}\right)\right\} e_{1}+\left\{e_{1} b-e_{2} c+b \omega_{2}^{1}\left(e_{2}\right)\right\} e_{2} .
\end{aligned}
$$


Assume that $M$ is biharmonic. It follows from (4.10) and (4.15) that

$$
\begin{array}{r}
-e_{1} c+e_{2} b+a \omega_{1}^{2}\left(e_{2}\right)=0, \\
e_{1} b-e_{2} c+b \omega_{2}^{1}\left(e_{2}\right)=0 .
\end{array}
$$

Combining (4.13), (4.14), (4.16) and (4.17), we have

$$
c \omega_{1}^{2}\left(e_{2}\right)=b \omega_{1}^{2}\left(e_{2}\right)=0 .
$$

If $\omega_{1}^{2}\left(e_{2}\right) \neq 0$ on an open subset $U$, then $b=c=0$ and hence $a=0$ on $U$ from (4.16). However, it contradicts the fact that $\langle H, H\rangle=\frac{(a+c)^{2}}{4}>0$. Therefore $\omega_{1}^{2}\left(e_{2}\right)=0$ on $M$. Consequently, we get

$$
\omega_{1}^{2}=0
$$

Thus the relation (4.11) is reduced to

$$
\begin{aligned}
\Delta H & =h\left(e_{1}, A_{H} e_{1}\right)-h\left(e_{2}, A_{H} e_{2}\right) \\
& =\frac{(a+b)}{2}\left\{\left(a^{2}-2 b^{2}+c^{2}\right) J e_{1}+b(a+c) J e_{2}\right\} .
\end{aligned}
$$

By (4.20) and (4.10) we see that

$$
\begin{gathered}
b=0, \\
a^{2}+c^{2}=5 \epsilon .
\end{gathered}
$$

Moreover, by (4.9) we have

$$
a c-c^{2}+\epsilon=0 .
$$

Case (II): $\langle H, H\rangle<0$. In this case, $c=-a$. From (4.6) and (4.8), we obtain

$$
-e_{1}(b-d)=(b-d) \omega_{1}^{2}\left(e_{2}\right) .
$$

Since $\langle H, H\rangle=-\frac{(b-d)^{2}}{4}=$ constant, we have $\omega_{1}^{2}\left(e_{2}\right)=0$. It follows that (4.6)-(4.8) is reduced to

$$
\begin{gathered}
e_{2} a=-e_{1} b-3 a \omega_{1}^{2}\left(e_{1}\right), \\
e_{2} b=-e_{1} a-(2 b+d) \omega_{1}^{2}\left(e_{1}\right) .
\end{gathered}
$$

As for the component of $\Delta H$ tangential to $M$, we have

$$
\begin{aligned}
\frac{1}{|H|}(\Delta H)^{\top}= & A_{D_{e_{1}} J e_{2}} e_{1}+\nabla_{e_{1}}\left(A_{2} e_{1}\right)-A_{2}\left(\nabla_{e_{1}} e_{1}\right)-A_{D_{e_{2}} J e_{2}} e_{2}-\nabla_{e_{2}}\left(A_{2} e_{2}\right)+A_{2}\left(\nabla_{e_{2}} e_{2}\right) \\
= & \omega_{2}^{1}\left(e_{1}\right) A_{1} e_{1}+\nabla_{e_{1}}\left(-b e_{1}-a e_{2}\right)-\omega_{1}^{2}\left(e_{1}\right) A_{2} e_{2}-\nabla_{e_{2}}\left(a e_{1}+d e_{2}\right) \\
= & \omega_{2}^{1}\left(e_{1}\right)\left(a e_{1}+b e_{2}\right)-\left(e_{1} b\right) e_{1}-b \omega_{1}^{2}\left(e_{1}\right) e_{2}-\left(e_{1} a\right) e_{2} \\
& -a \omega_{2}^{1}\left(e_{1}\right) e_{1}-\omega_{1}^{2}\left(e_{1}\right)\left(a e_{1}+d e_{2}\right)-\left(e_{2} a\right) e_{1}-\left(e_{2} d\right) e_{2} \\
= & -\left\{e_{1} b+e_{2} a+a \omega_{1}^{2}\left(e_{1}\right)\right\} e_{1}-\left\{e_{1} a+e_{2} d+d \omega_{1}^{2}\left(e_{1}\right)\right\} e_{2} .
\end{aligned}
$$


We suppose that $M$ is biharmonic. By (4.10) and (4.27), we have

$$
\begin{aligned}
& e_{1} b+e_{2} a+a \omega_{1}^{2}\left(e_{1}\right)=0 \\
& e_{1} a+e_{2} d+d \omega_{1}^{2}\left(e_{1}\right)=0 .
\end{aligned}
$$

Combining (4.25), (4.26), (4,28) and (4.29), we get

$$
a \omega_{1}^{2}\left(e_{1}\right)=b \omega_{1}^{2}\left(e_{1}\right)=0 .
$$

By the similar arguments as the case of $\langle H, H\rangle>0$, we obtain

$$
\omega_{1}^{2}=0 \text {. }
$$

Thus, the relation (4.11) is reduced to

$$
\begin{aligned}
\Delta H & =h\left(e_{1}, A_{H} e_{1}\right)-h\left(e_{2}, A_{H} e_{2}\right) \\
& =\frac{(a+b)}{2}\left\{a(b-d) J e_{1}-\left(b^{2}-2 c^{2}+d^{2}\right) J e_{2}\right\} .
\end{aligned}
$$

By (4.32), (4.10) and (4.9), we see that

$$
\begin{gathered}
a=0, \\
-b^{2}-d^{2}=5 \epsilon, \\
b^{2}+b d+\epsilon=0 .
\end{gathered}
$$

From (4.22) and (4.34) we can easily obtain the following proposition.

Proposition 3. There exist no proper biharmonic Lagrangian surfaces of constant mean curvature satisfying $\langle H, H\rangle>0$ (resp. $<0)$ in $\tilde{M}_{1}^{2}(4 \epsilon)$ with $\epsilon<0$ (resp. $\left.>0\right)$.

Next, we apply a construction method from [14] in order to obtain the explicit representation of the immersions of (I) and (II) when the ambient spaces are $\tilde{M}_{1}^{2}(4)=$ $\mathbf{C} P_{1}^{2}(4)$ and $\tilde{M}_{1}^{2}(-4)=\mathbf{C} H_{1}^{2}(-4)$, respectively. Let $\mathbf{C}_{s}^{3}$ be the complex 3-space with the complex coordinates $\left(z_{1}, z_{2}, z_{3}\right)$, whose metric is given by $g_{s, 3}(z, w):=\operatorname{Re}\left(\sum_{i=1}^{3-s} z_{i} \bar{w}_{i}-\right.$ $\left.\sum_{j=4-s}^{3} z_{j} \bar{w}_{j}\right)$. We consider the differential manifolds: $S_{2}^{5}(1)=\left\{z \in \mathbf{C}_{1}^{3} \mid g_{1,3}(z, z)=1\right\}$ and $H_{3}^{5}(-1)=\left\{z \in \mathbf{C}_{2}^{3} \mid g_{2,3}(z, z)=-1\right\}$. Hopf fibrations $\pi: S_{2}^{5}(1) \rightarrow \mathbf{C} P_{1}^{2}(4)$ and $\pi:$ $H_{3}^{5}(-1) \rightarrow \mathbf{C} H_{1}^{2}(-4)$ are defined as

$$
z \rightarrow z \cdot \mathbf{C} .
$$

Namely $\pi$ maps a vector $z$ to a complex line $z \mathbf{C}$.

Every Lagrangian immersion $f: M_{1}^{2} \rightarrow \mathbf{C} P_{1}^{2}(4)\left(\right.$ resp. $\left.f: M_{1}^{2} \rightarrow \mathbf{C} H_{1}^{2}(-4)\right)$ can be lifted locally to a Legendrian immerison $\tilde{f}: M_{1}^{2} \rightarrow S_{2}^{5}(1)$ (resp. $\tilde{f}: M_{1}^{2} \rightarrow H_{3}^{5}(-1)$ ). Here a Legendrian immersion $\tilde{f}$ is an immersion satisfying if $(p) \perp f_{\star}\left(T M_{1}^{2}\right)$ for all $p \in M_{1}^{2}$, where $i=\sqrt{-1}$. Conversely, let $\tilde{f}: M_{1}^{2} \rightarrow S_{2}^{5}(1)$ (resp. $\tilde{f}: M_{1}^{2} \rightarrow H_{3}^{5}(-1)$ ) be a Legendrian immersion. Then $\pi(\tilde{f}): M_{1}^{2} \rightarrow \mathbf{C} P_{1}^{2}(4)$ (resp. $M_{1}^{2} \rightarrow \mathbf{C} H_{1}^{2}(-4)$ ) is a Lagrangian immersion.

Let $M_{1}^{2}$ be a Legendrian surface of $S_{2}^{5}(1) \in \mathbf{C}_{1}^{3}$ or of $H_{3}^{5}(-1) \in \mathbf{C}_{2}^{3}$. Denote the Levi-Civita connection of $\mathbf{C}_{s}^{3}$ by $\bar{\nabla}$. Let $\bar{h}$ be the second fundamental form of $M_{1}^{2}$ in 
$S_{2}^{5}(1)$ or in $H_{3}^{5}(-1)$. Then we have $\pi_{\star} \bar{h}=h$ and

$$
\bar{\nabla}_{X} Y=\nabla_{X} Y+\bar{h}(X, Y)-\tilde{\epsilon}\langle X, Y\rangle \tilde{f},
$$

where $\tilde{\epsilon}=1$ or -1 depending on the ambient space is $\mathbf{C}_{1}^{3}$ or $\mathbf{C}_{2}^{3}$.

THEOREM 4. (I) Proper biharmonic Lagrangian surfaces of constant mean curvature in $\mathbf{C} P_{1}^{2}(4)$ are locally given by

$$
\begin{gathered}
f(x, y)=\pi\left(\sqrt{\frac{c^{2}}{c^{2}+1}} e^{-\frac{i}{c} x}, \sqrt{\frac{1}{c^{2}+1}} e^{i c x} \cosh \sqrt{c^{2}+1} y\right. \\
\left.\sqrt{\frac{1}{c^{2}+1}} e^{i c x} \sinh \sqrt{c^{2}+1} y\right)
\end{gathered}
$$

where $c=\frac{\sqrt{7 \pm \sqrt{41}}}{2}$.

(II) Proper biharmonic Lagrangian surfaces of constant mean curvature in $\mathbf{C H} H_{1}^{2}(-4)$ are locally given by

$$
\begin{aligned}
f(x, y)= & \pi\left(\sqrt{\frac{1}{b^{2}+1}} e^{-i b y} \sinh \sqrt{b^{2}+1} x, \sqrt{\frac{1}{b^{2}+1}} e^{-i b y} \cosh \sqrt{b^{2}+1} x,\right. \\
& \left.\sqrt{\frac{b^{2}}{b^{2}+1}} e^{\frac{i}{b} y}\right),
\end{aligned}
$$

where $b=\frac{\sqrt{7 \pm \sqrt{41}}}{2}$.

Proof. In case (I), it follows from (4.1), (4.19) and (4.21) that a Legendrian lift $\tilde{f} \in \mathbf{C}_{1}^{3}$ satisfies the PDE system

$$
\begin{gathered}
\tilde{f}_{x x}=a i \tilde{f}_{x}-\tilde{f}, \\
\tilde{f}_{y y}=-c i \tilde{f}_{x}+\tilde{f}, \\
\tilde{f}_{x y}=c i \tilde{f}_{y} .
\end{gathered}
$$

By replacing $x$ by $-x$ if necessary, we may assume that $c \geq 0$. Solving (4.39)-(4.41) and using $\langle\tilde{f}, \tilde{f}\rangle=1,\left\langle\tilde{f}_{x}, \tilde{f}_{y}\right\rangle=\left\langle\tilde{f}_{x}, i \tilde{f}_{y}\right\rangle=0,\left\langle\tilde{f}_{x}, \tilde{f}_{x}\right\rangle=1,\left\langle\tilde{f}_{y}, \tilde{f}_{y}\right\rangle=-1$, (4.22) and (4.23), we find that the immersion is congruent to (4.37) with one of $c$ 's. Here we note that proper biharmonic Lagrangian surfaces defined by two real solutions $c$ 's are noncongruent each other, because if two Lagrangian surfaces $\pi(\tilde{f})$ and $\pi(\tilde{g})$ are congruent, they must satisfy $\left\langle\tilde{g}_{x y},-i \tilde{g}_{y}\right\rangle=\left\langle\tilde{f}_{x y},-i \tilde{f}_{y}\right\rangle=c$ by (4.41).

In case of (II), by (4.1), (4.31) and (4.33) we see that a Legendrian lift $\tilde{f} \in \mathbf{C}_{2}^{3}$ satisfies the following PDE system:

$$
\begin{gathered}
\tilde{f}_{x x}=b i \tilde{f}_{y}+\tilde{f} \\
\tilde{f}_{y y}=d i \tilde{f}_{y}-\tilde{f}, \\
\tilde{f}_{x y}=-b i \tilde{f}_{x} .
\end{gathered}
$$

Similar to case (I), the immersion is congruent to (4.38) with one of $b$ 's. 
Case (III): $\langle H, H\rangle=0$ and $H \neq 0$. In this case, the author [16] proved that $M$ is biharmonic if and only if $\epsilon=0$ and $\Delta H=0$, however this fact is not enough information for classification of such surfaces. So, we need to investigate necessary and sufficient conditions for $M$ to be biharmonic more precisely. For this reason, we shall compute $\Delta H$ in detail (cf. [16]).

The mean curvature vector field is given by

$$
H=\alpha\left(J e_{1}+J e_{2}\right)
$$

for some function $\alpha$. Hence, $2 H=\sum_{i=1}^{2}\left\langle e_{i}, e_{i}\right\rangle h\left(e_{i}, e_{i}\right)$ implies

$$
2 \alpha=a+c=b-d .
$$

From Proposition 3 in [16],

$$
\Delta^{D} H=-G H
$$

REMARK 5. In [16], the equation $\Delta^{D} H=G H$ is derived. But this is incorrect. This error is not serious for the forthcoming proof in this paper.

On the other hand, by substituting $d=a-b-c$ into (4.9),

$$
G=(a-c-2 b)(c-b)+\epsilon .
$$

Hence we have

$$
\Delta^{D} H=\{-(a-c-2 b)(c-b)-\epsilon\} H .
$$

Let $(\Delta H)^{\perp}$ be the normal part of $\Delta H$. Then by using (4.11), (4.47) and (4.49) we obtain

$$
\begin{aligned}
(\Delta H)^{\perp}= & -G H+h\left(e_{1}, A_{H} e_{1}\right)-h\left(e_{2}, A_{H} e_{2}\right) \\
= & \alpha\left\{(a-c-2 b)(b-c)-\epsilon+\left(a^{2}-2 b^{2}+c^{2}-a b-2 b c+c d\right)\right\} J e_{1} \\
& +\alpha\left\{(a-c-2 b)(b-c)-\epsilon+\left(2 c^{2}-b^{2}-d^{2}+a b+2 b c-c d\right)\right\} J e_{2} .
\end{aligned}
$$

Furthermore, substituting $d=b-a-c$ into the right hand side of (4.50) we have

$$
\begin{aligned}
(\Delta H)^{\perp}= & \alpha\{(a+2 b-c)(a-c-2 b)-\epsilon\} J e_{1} \\
& +\alpha\{(-a+2 b-3 c)(a-c-2 b)-\epsilon\} J e_{2} .
\end{aligned}
$$

Assume that $M$ is biharmonic. It follows from (4.10) and (4.51) that

$$
a-c=2 b \text {. }
$$

Hence, (4.51) implies that

$$
(\Delta H)^{\perp}=-\epsilon H .
$$

By combining (4.10) and (4.53) we obtain

$$
\epsilon=0 \text {. }
$$

Conversely, suppose that $a-c=2 b$ and $\epsilon=0$. 
LEMMA 6. If $a-c=2 b$, then we have the following.

$$
\begin{gathered}
\nabla_{e_{i}}\left(A_{H} e_{i}\right)=\left\langle e_{i}, e_{i}\right\rangle\left\{e_{i} \alpha^{2}+\alpha^{2} \omega_{1}^{2}\left(e_{i}\right)\right\}\left(e_{1}+e_{2}\right), \\
A_{H}\left(\nabla_{e_{i}} e_{i}\right)=-\left\langle e_{i}, e_{i}\right\rangle \alpha^{2} \omega_{1}^{2}\left(e_{i}\right)\left(e_{1}+e_{2}\right), \\
A_{D_{e_{i}} H} e_{i}=\left\langle e_{i}, e_{i}\right\rangle \alpha\left\{e_{i} \alpha+\alpha \omega_{1}^{2}\left(e_{i}\right)\right\}\left(e_{1}+e_{2}\right),
\end{gathered}
$$

where $i=1,2$.

Proof. By using $a-b=b+c=-c-d=\alpha$ we can prove the lemma (cf. Lemma 8 of [16]).

By applying Lemma 6, we obtain

$$
\begin{aligned}
(\Delta H)^{\top} & =\nabla_{e_{1}}\left(A_{H} e_{1}\right)+A_{D_{e_{1}} H} e_{1}-\nabla_{e_{2}}\left(A_{H} e_{2}\right)-A_{D_{e_{2}} e_{2}}-A_{H}\left(\nabla_{e_{1}} e_{1}\right)+A_{H}\left(\nabla_{e_{2}} e_{2}\right) \\
& =3 \alpha\left\{e_{1} \alpha+e_{2} \alpha+\alpha \omega_{1}^{2}\left(e_{1}\right)+\alpha \omega_{1}^{2}\left(e_{2}\right)\right\}\left(e_{1}+e_{2}\right) .
\end{aligned}
$$

On the other hand, (4.6), (4.8) and (4.46) yield

$$
e_{1} \alpha+e_{2} \alpha=-\left(\omega_{1}^{2}\left(e_{1}\right)+\omega_{1}^{2}\left(e_{2}\right)\right)
$$

Combining (4.58) and (4.59) we get (cf. Proposition 9 of [16])

$$
(\Delta H)^{\top}=0 .
$$

Also, by (4.51), $a-c=2 b$ and $\epsilon=0$ we get $(\Delta H)^{\perp}=0$, and hence $\Delta H=(\Delta H)^{\top}+$ $(\Delta H)^{\perp}=0$. Consequently, we obtain the following lemma.

LEMMA 7. In the case when $M$ is marginally trapped, $M$ is biharmonic if and only if $\epsilon=0$ and $a-c=2 b$.

Therefore, by the proof of Proposition 10 of [16], we can show the following statement.

THEOREM 8. Let $M$ be a marginally trapped biharmonic Lagrangian surface in $\tilde{M}_{1}^{2}(4 \epsilon)$. Then $\epsilon=0$, and there exists a coordinate system $\{x, y\}$ defined in a neighborhood $U$ of $p \in M$ such that the metric tensor of $U$ is given by $g=d x^{2}-d y^{2}$ and the second fundamental form is given by

$$
\begin{gathered}
h\left(\partial_{x}, \partial_{x}\right)=a J \partial_{x}+\frac{a-c}{2} J \partial_{y}, \quad h\left(\partial_{y}, \partial_{y}\right)=-c J \partial_{x}-\frac{a+3 c}{2} J \partial_{y}, \\
h\left(\partial_{x}, \partial_{y}\right)=-\frac{a-c}{2} J \partial_{x}+c J \partial_{y}, \\
c=-\frac{x+y}{8}\left(\partial_{x}-\partial_{y}\right) f(x-y)+g(x-y), \quad a=f(x-y)-c .
\end{gathered}
$$

Conversely, suppose that $a$ and $c$ are functions on a simply-connected domain $U \subset$ $\mathbf{R}^{2}$ defined by (4.62). Let $g=d x^{2}-d y^{2}$ be the metric tensor on $U$. Then, up to rigid motions of $\mathbf{C}_{1}^{2}$, there exists a unique Lagrangian immersion of $(U, g)$ into $\mathbf{C}_{1}^{2}$ whose second fundamental form is given by (4.61). Moreover such a surface is marginally trapped biharmonic.

REMARK 9. After this paper was completed, the author found out that the position vectors of Lagrangian surfaces in Theorem 8 were obtained explicitly by Chen and Dillen as follows (see, (i.2) in Theorem 4.1 of [8]): 
$L(s, t)=c_{1} s e^{i f(t)}+z(t)$, where $f(t)$ is a real-valued function, $c_{1}$ is a light-like vector, and $z(t)$ is a null curve in $\mathbf{C}_{1}^{2}$ satisfying $\left\langle i z^{\prime}(t), c_{1} e^{i f(t)}\right\rangle=0$ and $\left\langle z^{\prime}(t), c_{1} e^{i f(t)}\right\rangle=-1$. Here, a curve $z$ is called null if it satisfies $\left\langle z^{\prime}, z^{\prime}\right\rangle=0$. We can easily see that $L$ is biharmonic.

REMARK 10. In [6], marginally trapped biharmonic surfaces of real index 1 of $\mathbf{C}_{1}^{2}$ were classified. But this classification was not complete. In fact, marginally trapped biharmonic surfaces in [6] satisfy $A_{H}=0$, however, surfaces in Theorem 8 of this paper do not satisfy $A_{H}=0$, and hence they are new examples of marginally trapped biharmonic surfaces in $\mathbf{C}_{1}^{2}$. result.

By the similar computations as the proof in Theorem 4, we have the following

THEOREM 11. Let $M^{2}$ be a proper biharmonic Lagrangian surface of constant mean curvature in $\tilde{M}^{2}(4 \epsilon)$. Then $\epsilon>0$ and proper biharmonic Lagrangian surfaces of constant mean curvature in $\mathbf{C} P^{2}(4)$ are locally given by

$$
\begin{gathered}
f(x, y)=\pi\left(\sqrt{\frac{c^{2}}{c^{2}+1}} e^{-\frac{i}{c} x}, \sqrt{\frac{1}{c^{2}+1}} e^{i c x} \cos \sqrt{c^{2}+1} y\right. \\
\left.\sqrt{\frac{1}{c^{2}+1}} e^{i c x} \sin \sqrt{c^{2}+1} y\right)
\end{gathered}
$$

where $c=\frac{\sqrt{7 \pm \sqrt{41}}}{2}$ and $\pi: S^{5}(1) \rightarrow \mathbf{C} P^{2}(4)$ is the Hopf fibration.

\section{REFERENCES}

1. M. Barros and A. Romero, Indefinite Kaehler manifolds, Math. Ann. 261 (1982) 55-62.

2. R. Caddeo, S. Montaldo and C. Oniciuc, Biharmonic submanifolds of $S^{3}$, Internat. J. Math. 12 (2001), 867-876.

3. R. Caddeo, S. Montaldo and C. Oniciuc, Biharmonic submanifolds in spheres, Israel J. Math. 130 (2002), 109-123.

4. B.-Y. Chen, Null 2-type surfaces in Euclidean space, in Algebra, analysis and geometry (Taipei, 1988), (World Sci. Publ., Teaneck, NJ, 1989), 1-18.

5. B.-Y. Chen, Riemannian geometry of Lagrangian submanifolds, Taiwanese J. Math. 5 (2001) 681-723.

6. B.-Y. Chen and S. Ishikawa, Biharmonic pseudo-Riemannian submanifolds in pseudoEuclidean spaces, Kyushu J. Math. 52 (1998) 167-185.

7. B.-Y. Chen and L. Vrancken, Lagrangian minimal isometric immersion of a Lorentzian real space form into a Lorentzian complex space form, Tohoku Math. J. 54 (2002) 121-143.

8. B.-Y. Chen and F. Dillen, Classification of marginally trapped Lagrangian surfaces in Lorentzian complex space forms, J. Math. Phys., to appear.

9. J. Eells and J. H. Sampson, Variational theory in fiber bundles, in Proc. U.S.-Japan seminar in differential geometry (Kyoto, 1965), 22-33.

10. J. Inoguchi, Submanifolds with harmonic mean curvature vector field in contact 3manifolds, Colloq. Math. 100 (2004), 163-179.

11. G. Y. Jiang, 2-harmonic maps and their first and second variational formulas. (Chinese), Chinese Ann. Math. A 7 (1986), 389-402.

12. M. Kriele and L. Vrancken, Minimal Lagrangian submanifolds of Lorentzian complex space forms with constant sectional curvature, Arch. Math. (Basel) 72 (1999), 223-232. 
13. S. Montaldo and C. Oniciuc A short survey on biharmonic maps between Riemannian manifolds, Rev. Un. Mat. Argentina 47 (2006), no. 2, 1-22. Available at http://inmabb.criba. edu.ar/revuma/

14. H. Reckziegel, Horizontal lifts of isometric immersions into the bundle space of a pseudo-Riemannian submersion, in Global differential geometry and global analysis (Berlin, 1984), 264-279, Lecture Notes in Math., 1156, (Springer-Verlag, 1985).

15. T. Sasahara, Legendre surfaces in Sasakian space forms whose mean curvature vectors are eigenvectors, Publ. Math. Debrecen 67 (2005), 285-303.

16. T. Sasahara, Quasi-minimal Lagrangian surfaces whose mean curvature vectors are eigenvectors. Demonstratio Math. 38 (2005), 185-196.

17. L. Vrancken, Minimal Lagrangian submanifolds with constant sectional curvature in indefinite complex space forms, Proc. Amer. Math. Soc. 130 (2002), 1459-1466. 